\title{
A sintomatologia do stress sob a ótica da Medicina Tradicional Chinesa
}

\section{The symptoms of stress from the perspective of Traditional Chinese Medicine}

Rodrigo Almeida Bastos ${ }^{1}$

${ }^{1}$ Programa de Pós-Graduação em Psicologia, Universidade Federal de Santa Maria (UFSM) - Santa Maria (RS), Brasil.

DOI: http://dx.doi.org/10.7322/abcshs.v40i2.738

\section{RESUMO}

O desequilíbrio propiciado pelo stress desencadeia uma série de prejuízos aos diversos setores da vida dos indivíduos. A presença de stress pode desencadear o desenvolvimento de inúmeras doenças e propiciar um prejuízo para a qualidade de vida e produtividade do ser humano. A Medicina Tradicional Chinesa pode ser instrumento eficaz de avaliação e tratamento da presença do stress por visar à reorganização homeostática do organismo. Baseando-se na manutenção da qualidade de vida do ser humano, o presente estudo objetivou realizar uma revisão bibliográfica da sintomatologia do stress sob a ótica da Medicina Tradicional Chinesa. As relações encontradas entre a sintomatologia do stress e a Medicina Tradicional Chinesa foram: Vazio de Qi/Yang do Coração (Cansaço e Desgaste Físico), Estagnação do Qi do Fígado (Tensão Muscular), Fogo Fleuma do Coração, Estagnação do Qi do Coração e Fígado e Deficiência de Qi, Yin ou Sangue do Coração (Ansiedade/Angustia diária), Mucosidade-Fogo no Estômago e Coração (Pensamentos constantes em um só assunto), o Fogo no Coração que consome o Yin e Vazio de Sangue do Coração (Insônia). Estas inter-relações entre a Medicina Ocidental e a Medicina Tradicional Chinesa poderão ser importantes no direcionamento efetivo do tratamento da sintomatologia do stress.

Palavras-chave: Medicina Tradicional Chinesa; estresse fisiológico; estresse mecânico; estresse psicológico.

\begin{abstract}
The imbalance brought by stress triggers a series of burdens in various sectors of an individual's life. The presence of stress can trigger the development of numerous diseases and cause a detriment to the quality of life and productivity of human beings. Traditional Chinese Medicine can be an effective tool to evaluate and treat stress symptoms, as it aims at the homeostatic reorganization of the body. This study aimed to conduct a literature review of stress symptoms from the perspective of the Traditional Chinese Medicine, based in maintenance of quality of life. The observed relations between stress symptoms and Traditional Chinese Medicine were Heart Qi/Yang Deficiency (Physical Fatigue and Wear), Liver Qi Stagnation (Muscle Tension), Heart Phlegm Fire, Heart and Liver Qi Stagnation and Heart Qi/Yin/Blood Deficiency (Daily Anxiety/Anguish), Mucus-Fire in Stomach and Heart (Repetitive thoughts), Heart Fire that consumes the Yin and Heart Blood Emptiness (Insomnia). Such interrelationships between western medicine and Traditional Chinese Medicine can be important in treatment of the symptoms of stress.
\end{abstract}

Keywords: Chinese Traditional Medicine; physiological stress; mechanical stress; psychological stress. 


\section{INTRODUÇÃO}

O stress é uma reação orgânica que possui componentes físicos e/ou psicológicos, causado pelas alterações psicofisiológicas que ocorrem quando a pessoa enfrenta situações que a irrite, amedronte, excite ou confunda, ou mesmo lhe proporcione felicidade ${ }^{1}$. Deste modo, o stress é um estado de tensão mental e físico que produz um desequilíbrio no funcionamento global do ser humano.

O termo stress recebeu sua significância no âmbito da saúde pela primeira vez com Hans Selye em $1926^{2}$. Durante o atendimento a seus pacientes, Selye identificou que todos os indivíduos expostos a uma ameaça produziam mecanismos de defesa que se assemelhavam entre si. Eram sintomas como dores nas articulações, problemas digestivos, falta de apetite, febre, hepato e esplenomegalia e patologias dermatológicas, que não podiam caracterizar uma doença especifica ${ }^{3}$.

Suas experiências em ratos mostraram que se o organismo animal for exposto a agentes não específicos agudos, tais como a exposição ao frio ou exercício muscular excessivo, sintomas típicos aparecem. Os sintomas são independentes da natureza do agente prejudicial ou do tipo farmacológico da droga empregada, e se apresentaram como uma resposta aos danos ${ }^{3}$. Em estudos que correlacionaram estes dados aos humanos ${ }^{4}$, observou-se que as demandas de caráter social e as ameaças do ambiente no qual o indivíduo estava inserido requeriam capacidade de adaptação, provocando a presença do stress.

Diante dos sintomas típicos acerca do esforço generalizado do organismo em se adaptar a novas circunstâncias ${ }^{5}$, descriminou-se a "Síndrome Geral da Adaptação" (SAG). Desta forma, foram descritas três fases para a presença do stress: Reação de Alarme, Fase de Resistência e Fase de Exaustão.

Na reação de alarme, o organismo está enfrentando uma situação a qual se sente ameaçado, provocando o que se chama de quebra da homeostase, ou quebra do equilíbrio orgânico interno. A sintomatologia é notada de forma a ajudar o organismo neste primeiro momento, não sendo prejudicial à saúde do individuo ${ }^{6}$. Se não for excessivo, na fase Alarme o indivíduo encontra motivação, entusiasmo, energia e produtividade ${ }^{6,7}$.

A fase de resistência ocorre quando o estressor perdura por um período muito prolongado. Nessa fase, o organismo tenta readquirir a homeostasia e utiliza suas reservas de energia adaptativa. É nesta fase que o individuo pode readquirir o equilíbrio interno sem maiores danos. Assim, os sintomas da primeira fase tendem a cessar ${ }^{6}$. Havendo persistência dos fatores estressores em frequência ou intensidade, haverá uma quebra na resistência do organismo e ele passa à fase de "quase-exaustão". A observação desta fase permitiu a ampliação o sistema trifásico para uma sistematização quadrifásica do stress $^{8}$. Nesta fase, o processo de adoecimento se inicia e não apenas a resistência orgânica, mas também a mental mostra sinais de desgaste. A depressão passa a fazer parte do quadro de sintomas, que se prolongam na "fase de exaustão" caso não haja remoção dos estressores ou uso de estratégias de enfrentamento.
Porém, quando o organismo não consegue adaptar-se ou mesmo, como o próprio nome sugere, tenta resistir aos fatores estressores, o processo de stress pode desenvolver-se até o ponto mais crítico, que é fase de exaustão $0^{6,7}$.

Nesta fase de Exaustão, o organismo já não tem energia para se adaptar à situação de stress, tornando-se mais exposto aos agentes estressores. O stress torna-se mais intenso e, em consequência, faz esgotar a energia adaptativa do organismo ${ }^{6}$.

$\mathrm{O}$ estado prolongado de stress pode interferir no bem-estar psicológico e na qualidade de vida das pessoas ${ }^{9,10}$. O stress, se gerenciado de forma eficaz, propicia um ambiente social, familiar e laboral saudável e produtivo ${ }^{11-14}$.

Visto que existe a quebra da homeostase orgânica e psicológica de um indivíduo, a análise do processo de stress por uma ciência que não trata a doença, e sim o padrão energético desarmônico que a causa, se faz relevante. Neste sentido, a Medicina Tradicional Chinesa (MTC), pode ser instrumento terapêutico valioso de avaliação e tratamento da presença de stress.

\section{Processo Terapêutico da Medicina Tradicional Chinesa}

A MTC toma como base a existência de uma estrutura energética integrada ao corpo físico, por onde a energia circula em canais, chamados meridianos. Os meridianos são vias que interligam os diversos sistemas energéticos do corpo, por onde circulam energias vital (Qi) ou patogênica (Xie), os quais agem diretamente sobre o organismo e abrigam pontos específicos. Estes pontos, ao serem estimulados, reorganizam a circulação energética de todo o corpo. A doença, por sua vez, é sempre a desorganização da energia funcional que controla e dinamiza os meridianos. Todo este sistema, que se desenvolveu de experimentação e aperfeiçoamento há milhares de anos, pode ser equilibrado através da Acupuntura ${ }^{15}$.

A base de tudo é o $Q i$, a força vital. Mas não se trata apenas da força vital do ser humano ou do animal, sendo essa uma energia universal que cerca e permeia todas as coisas, animadas e inanimadas, e que circula no organismo através dos meridianos. Os chineses, portanto, encaram todo o funcionamento do organismo e da mente como dependente do fluxo normal das energias do organismo ou da força vital, a que se denomina $Q i^{16}$.

Para a fisiologia ocidental, a Acupuntura é uma técnica que atua na modulação nervosa em três instâncias: local, espinal/segmentar ou supraespinal/suprasseguimentar. A visão da Medicina Tradicional Chinesa descreve a técnica como a estimulação dos pontos energéticos distribuídos pelos canais, baseado na teoria do Yin e Yang ${ }^{17}$. São usados como principais instrumentos: agulhas de acupuntura, dedos (acupressão), ventosa ou pelo aquecimento promovido por moxa (um bastão de Artemísia em brasa, que é aproximado à pele para aquecer o ponto de acupuntura), dentre outros ${ }^{15}$.

A Organização Mundial de Saúde ${ }^{18}$ tornou a Acupuntura uma referência de tratamento mundial. Essa organização emitiu uma lista em que reconhece a eficácia deste tratamento em diversas enfermidades. Entretanto, o diagnóstico, as avaliações e o 
tratamento devem ser realizados por um profissional com formação adequada e comprovada.

O desequilíbrio propiciado pelo stress desencadeia uma série de prejuízos nos diversos setores da vida dos indivíduos ${ }^{12-14}$. Além disso, o stress pode desencadear o desenvolvimento de inúmeras doenças e proporcionar um prejuízo para a qualidade de vida e produtividade do ser humano. Diante deste contexto, o presente estudo pretende ampliar o horizonte terapêutico para o tratamento do stress sob a ótica da Medicina Tradicional Chinesa.

\section{Descrição Sintomática da presença do stress}

Ao avaliar a gama situacional onde o stress poderia se desenvolver, observou-se, por frequência de casos, as prioridades de análise: no âmbito pessoal, onde o stress do sujeito surge por situações diversas do dia-a-dia; ocupacional, em que o ambiente laboral seria a fonte do stress; e em contexto patológico, verificando o stress em relação às doenças crônicas. Sendo assim, foi selecionado um artigo para cada uma das três prioridades de análise, a fim de descrever os sintomas de stress presentes em cada situação.

\section{O uso da acupuntura na sintomatologia do stress}

O trabalho realizado ${ }^{16}$, tendo como amostra uma população adulta em geral, teve como objetivo expandir as terapêuticas e os programas voltados ao controle do stress. Em relação aos sintomas mais assinalados, segundo a metodologia desta pesquisa, foram encontrados com predominância: sensação de desgaste físico constante (95\%), cansaço constante (90\%), tensão muscular (85\%) e ansiedade/angústia diária (85\%).

A média de idade dos sujeitos dessa pesquisa foi de 43,5 anos, com predominância de mulheres com ensino superior completo. No contexto profissional, a amostra apresentou pessoas teoricamente instruídas e/ou com conhecimento na área de saúde em busca de uma terapia complementar como forma de tratamento do stress.

Os autores observaram que a maioria dos participantes se encontrava na fase de resistência (60\%) e quase exaustão (35\%). O restante deles se encontrava na fase de alerta (5\%) e nenhum na fase de exaustão do stress. Após a intervenção com acupuntura, verificou-se nessa amostra uma redução dos sintomas de stress e da intensidade da queixa dos participantes. Sujeitos que, antes do tratamento, encontravam-se nas fases de alerta e resistência, após o tratamento, em sua maioria (75\%), não mais apresentavam stress, ou regrediram às fases iniciais de alerta (5\%) e resistência (20\%)

\section{Sintomas de estresse nos trabalhadores atuantes em cinco núcleos de saúde da família}

Este trabalho ${ }^{19}$ foi realizado com profissionais de saúde em um Centro de Saúde Escola da Universidade de São Paulo. Foi investigada uma amostra predominante de adultos jovens (40,5\% entre 19 e 29 anos), do sexo feminino (89\%). Os agentes comunitários de saúde constituem a maior parcela (54\%) da amostra, seguidos pelos auxiliares de enfermagem (19\%), e os médicos e enfermeiros têm a menor parcela $(13,5 \%)$.

Foi aplicado como instrumento de pesquisa o Inventário de Sintomas de Stress para adultos de Lipp que visa identificar a sintomatologia que o indivíduo apresenta, avaliando se ele possui sintomas de stress, o tipo de sintoma predominante e a fase em que se encontra. Verificou-se que 23 trabalhadores (62\%) encontravam-se em situação de stress: 19, na fase de resistência, 4 na fase de quase exaustão, e nenhum trabalhador foi encontrado na fase de alerta ou exaustão, de acordo com os sintomas apresentados. Neste contexto, identificou-se que $83 \%$ dos trabalhadores estressados possuem predominância de sintomas físicos e/ou psicológicos da fase de resistência, e 17\%, da fase de quase exaustão.

Identificadas as fases, o estudo organizou estatisticamente os sintomas prevalentes e concluiu que o sintoma físico mais referido na fase de resistência foi o "Desgaste Físico Constante", presente em $83 \%$ dos entrevistados. Em relação ao sintoma psicológico mais citado, encontrou-se "Pensar constantemente em um só assunto" (70\%).

$\mathrm{Na}$ fase de quase exaustão, o sintoma físico mais referido foi a "Insônia" (52\%) enquanto o sintoma psicológico prevalente foi a "Angustia/Ansiedade diária e vontade de fugir de tudo", referida por $65 \%$ dos sujeitos identificados como estressados.

Este estudo, em análise, sugere que a área psicológica de um indivíduo com stress é a mais afetada. Vale a pena ressaltar não apenas a existência do fator estressor, mas a percepção de cada profissional diante das situações, considerando que o trabalho direto com a comunidade é um fator estressor de grande magnitude e risco.

\section{Gênero e manifestação de stress em hipertensos}

A análise deste estudo ${ }^{20}$, permite investigar uma população de homens e mulheres adultos, em atividade laboral ou não, todos com diagnóstico de hipertensão arterial sistêmica. O estudo relata que $85,4 \%$ dos entrevistados $(n=88)$ apresentaram stress. Estes dados foram verificados a partir do Inventário de Sintomas de Stress para adultos, que avalia os sintomas e a fase do stress em cada indivíduo, e todos os valores foram estatisticamente significativos ${ }^{7}$.

Percebeu-se uma percentagem maior de homens na fase de resistência $(60,6 \%)$ enquanto as mulheres estavam em sua maioria na fase de quase exaustão, com percentil de 41,4\%. Em ambos os gêneros, os sintomas psicológicos predominaram nos indivíduos que se encontravam nas fases de Resistência, Quase-Exaustão e Exaustão (Tabela 1).

Os resultados analisados neste trabalho não apontam diferença significativa quanto à presença de stress entre homens e mulheres. Porém, é importante destacar que ambos os gêneros apresentaram desequilíbrios psicológicos maiores que os físicos na presença de stress e tendo a mesma condição patológica: Hipertensão Arterial Sistêmica. 


\section{Avaliação da sintomatologia do stress sob à ótica da Medicina Tradicional Chinesa}

O stress, na visão da Medicina Tradicional Chinesa, não se trata de uma doença, mas de um padrão de desequilíbrio energético ${ }^{21}$. Por não considerar a separação do corpo e da mente, mas um indivíduo de forma integral, a Medicina Tradicional Chinesa, que atua na ambiência física do paciente, agirá inexoravelmente também no estado psíquico. No entanto, a constituição genética de cada indivíduo irá definir qual sistema energético irá ser afetado primeiro no momento do stress $^{22,23}$.

Avaliando os trabalhos que descrevem os sintomas dos respectivos sujeitos expostos a diferentes fatores estressores, percebe-se a predominância de sintomas que serão analisados sob a ótica da Medicina Tradicional Chinesa (Tabela 2).

Pode-se observar uma pequena variação dos sintomas referidos pelos sujeitos das pesquisas analisadas. Segundo o Inventário de Sintomas de Stress para adultos de Lipp ${ }^{7}$, apenas um dos sintomas relatados pelos sujeitos das pesquisas faz parte da primeira fase de stress. Houve maior referência a sintomas da fase de Exaustão, onde o organismo já não tem condição de se adaptar ${ }^{6}$.

As emoções se desequilibram com a obstrução do fluxo de $Q i$ ou tornando-o irregular (excessos e deficiências) ${ }^{24,25}$. No presente estudo, foram encontrados sintomas causados pelo stress, que

Tabela 1: Frequência de sintomas de stress quanto ao gênero. Reproduzido de Wottrich et al. ${ }^{20}$

\begin{tabular}{|c|c|c|c|c|c|}
\hline & \multicolumn{2}{|c|}{ Homens } & \multicolumn{2}{|c|}{ Mulheres } & \multirow{2}{*}{$\begin{array}{c}\text { Valor } \\
\text { p }\end{array}$} \\
\hline & $n=33$ & $32 \%$ & $n=70$ & $68 \%$ & \\
\hline \multicolumn{6}{|l|}{ Fase Alerta } \\
\hline $\begin{array}{l}\text { Sintomas } \\
\text { físicos }\end{array}$ & 40,60 & 26,30 & 42,86 & 22,63 & 0,622 \\
\hline $\begin{array}{l}\text { Sintomas } \\
\text { psicológicos }\end{array}$ & 32,12 & 32,72 & 27,43 & 31,96 & 0,492 \\
\hline \multicolumn{6}{|c|}{ Fase Resistência e Quase-Exaustão } \\
\hline $\begin{array}{l}\text { Sintomas } \\
\text { físicos }\end{array}$ & 42,73 & 22,95 & 50,71 & 27,31 & 0,149 \\
\hline $\begin{array}{l}\text { Sintomas } \\
\text { psicológicos }\end{array}$ & 43,03 & 32,83 & 63,43 & 31,75 & 0,003 \\
\hline \multicolumn{6}{|l|}{ Fase Exaustão } \\
\hline $\begin{array}{l}\text { Sintomas } \\
\text { físicos }\end{array}$ & 32,75 & 19,13 & 34,65 & 19,42 & 0,643 \\
\hline $\begin{array}{l}\text { Sintomas } \\
\text { psicológicos }\end{array}$ & 38,21 & 27,72 & 54,46 & 31,93 & 0,014 \\
\hline
\end{tabular}

Tabela 2: Presença dos sintomas prevalentes, segundo fatores estressores e fases do stress

\begin{tabular}{|c|c|c|c|c|}
\hline \multirow[b]{2}{*}{ Sintomas } & \multicolumn{4}{|c|}{ Fator Estressor } \\
\hline & $\begin{array}{c}\text { Estressor } \\
\text { Geral }\end{array}$ & $\begin{array}{l}\text { Estressor } \\
\text { Ocupacional }\end{array}$ & $\begin{array}{l}\text { Estressor } \\
\text { Patológico }\end{array}$ & $\begin{array}{l}\text { Fase do } \\
\text { Stress }\end{array}$ \\
\hline Desgaste físico & $\mathrm{x}$ & $\mathrm{X}$ & X & Resistência \\
\hline Cansaço & $\mathrm{X}$ & & $\mathrm{X}$ & Resistência \\
\hline $\begin{array}{l}\text { Tensão } \\
\text { muscular }\end{array}$ & $x$ & & & Alerta \\
\hline $\begin{array}{l}\text { Ansiedade/ } \\
\text { Angústia diária }\end{array}$ & $\mathrm{x}$ & $\mathrm{x}$ & & Exaustão \\
\hline $\begin{array}{l}\text { Pensamentos } \\
\text { constantes em } \\
\text { um só assunto }\end{array}$ & & $\mathrm{x}$ & & Exaustão \\
\hline Insônia & & $\mathrm{x}$ & $\mathrm{X}$ & Exaustão \\
\hline
\end{tabular}

se caracterizam pela desarmonia do $Q i$, os quais serão descritos abaixo:

\section{Sintoma 1: Desgaste Físico e Cansaço}

Discriminado na fase de Resistência do stress e encontrado na maioria dos sujeitos analisados neste estudo, o cansaço e o desgaste físico podem ser diferenciados em Deficiência, com falta de energia, e Estagnação, na qual a energia está presente, porém bloqueada ${ }^{25}$.

O sintoma de Vazio de Qi/Yang do Coração é descrito ${ }^{15}$ como aquele que se expressa através do que se chamou de Fadiga Psicossomática, onde existe uma defasagem da circulação sanguínea, consequentemente havendo má nutrição e perfusão tecidual. É causado por um processo de stress que pode causar fraqueza constitucional ou Desgaste Físico e Cansaço.

\section{Sintoma 2: Tensão muscular}

Sintoma integrante da primeira fase do stress, a tensão muscular pode ser definida como Estagnação do $Q i$ do Fígado ${ }^{15}$, que prejudica o livre fluxo da energia corporal, desequilibrando o funcionamento dos músculos e outros sistemas orgânicos e energéticos. Pode gerar um esgotamento seguido por dores localizadas e/ou difusas, que não melhoram com a pressão. Esta definição vem ao encontro da relação entre Tensão Muscular e Eletricidade Estática ${ }^{26}$, onde existe um vazio elétrico a nível celular ocasionado pelo stress, seguido de dificuldade da circulação sanguínea, e o resultado final é um acumulo de toxinas nos músculos, causando a rigidez.

\section{Sintoma 3: Ansiedade/Angustia diária}

A ansiedade e a angústia denotam um estado de tensão ${ }^{25}$. A análise dos artigos selecionados permite a este estudo sugerir que os sujeitos que apresentaram a ansiedade como sintoma do stress o fizeram em fase de Exaustão, visto que o sintoma tem frequência diária e constante ${ }^{7}$.

Em estudos ${ }^{25}$, foram descritos os sintomas, na perspectiva da Medicina Tradicional Chinesa, relacionados à Ansiedade, a partir de três processos: Excesso, Estase ou Deficiência de sistemas energéticos. Como sintoma principal relacionado ao Excesso, descreveu-se o Fogo Fleuma do Coração. Segundo o autor, esta é uma forma de excesso na MTC que pode levar a uma confusão de pensamentos, linguagens e comportamentos. No processo de Estase ou Estagnação energética, descreveu-se a Estagnação do Q $i$ do Coração e Fígado como sintoma mais envolvido com a estagnação emocional causada pelo stress $^{25}$. Segundo a MTC, este sintoma leva à hiperatividade do sistema energético do fígado e distúrbios no sistema do coração, levando à Ansiedade e Angústia. Por fim, a Ansiedade pode estar relacionada à Deficiência energética, representada pelos sintomas Deficiência de Qi, Yin ou Sangue do Coração ${ }^{25}$. Quaisquer destes três sintomas causam desordem no espírito do coração (estado mental do indivíduo), já que são três elementos fundamentais para a manutenção da estabilidade mental. 


\section{Sintoma 4: Pensamentos constantes em um só assunto}

O sintoma foi encontrado em indivíduos na fase de Exaustão devido ao Stress Ocupacional, segundo a análise dos artigos apresentados neste estudo. Não se trata de um simples sintoma relacionado a preocupações cotidianas ${ }^{27}$. Existe importante diferença entre o pensamento determinado ou direcionado à resolução de uma situação e a ruminação de pensamentos relacionada à preocupação acerca desta.

Diversos autores descrevem que a personalidade de um sujeito que tende a manter pensamentos constantes de forma desequilibrada tende a pensar constantemente em trabalho, construindo junto aos resultados deste estudo a relação entre o Stress Ocupacional e o Pensamentos constantes em um só assunto ${ }^{24,25,27}$. Especialistas descrevem este desequilíbrio como Mucosidade-Fogo no Estômago e Coração ${ }^{15,25}$, no qual ocorre inquietação mental e perda no direcionamento dos pensamentos. Este sintoma energético tem relação direta com síndromes que afetam os sistemas responsáveis por clarear a mente e por promover o livre fluxo de energia, incluindo os pensamentos. É um padrão mental e emocional, que pode provocar sensação de agitação torácica e comportamento maníaco.

\section{Sintoma 5: Insônia}

A Insônia foi relatada pelos sujeitos expostos a estressores ocupacionais e patológicos e descrita como um sintoma de Exaustão ${ }^{7}$. A gravidade da insônia ocasionada por um processo de stress se mensura através de sua frequência. Seu estágio mais preocupante é descrito ao perceber que o sintoma ocorreu "sempre" ou "quase sempre" nas últimas duas semanas antes da entrevista do sujeito da pesquisa ${ }^{28}$.

Para a Medicina Chinesa, a insônia está relacionada a um distúrbio do "Espírito do Coração", denominado Shen; um conceito tradicional muito próximo do que a ciência ocidental chama de "Mente". Neste sentido, etiologicamente, pode-se afirmar que a síndrome energética relacionada à Insônia se assemelha à relacionada à Ansiedade ${ }^{25}$.

Esta síndrome energética tem, entre seus fatores etiopatogênicos, o "ferimento interno provocado pelas emoções". É uma descrição subjetiva, construída pela literatura especializada ${ }^{15}$ em referência à desarmonia orgânica provocada pelo stress no indivíduo. Este sintoma energético foi denominado de Vazio do Yin do Coração. Complementando este diagnóstico, descreve-se o Fogo no Coração provocado pelos estressores como fonte deste Vazio do $\mathrm{Yin}^{25}$. Sendo assim, a síndrome principal para o distúrbio orgânico da insônia é descrita como Fogo no Coração que Consome o Yin.

Ainda relacionando a insônia a um desequilíbrio do Shen, outra relação sintomática pode ser percebida, desta vez associada ao Sangue. A Medicina Chinesa destaca que o Sangue é a morada da mente, sendo governado pelo Coração. Sua função energética principal é carregar ao organismo um conjunto diverso de matéria nutriente ${ }^{15,25}$. Nesse sentido, a Medicina Chinesa observa que a deficiência de Sangue no Coração poderá acarretar em empobrecimento nutricional, desequilibrando a relação homeostática entre o Shen e o corpo, causando insônia e sono pouco reparador. Este desequilíbrio é chamado na literatura tradicional de Vazio de Sangue do Coração.

\section{CONCLUSÃO}

A partir dos artigos apresentados no presente estudo, é possível observar os sintomas mais frequentes em sujeitos expostos a fatores estressores variados e correlacioná-los à fisiologia energética de suas síndromes, segundo a Medicina Tradicional Chinesa. Essa correlação energética, como descrita, Vazio de Qi/Yang do Coração (Cansaço e Desgaste Físico); Estagnação do Qi do Fígado (Tensão Muscular); Fogo Fleuma do Coração, Estagnação do Qi do Coração e Fígado e Deficiência de Qi, Yin ou Sangue do Coração (Ansiedade/Angustia diária); Mucosidade-Fogo no Estômago e Coração (Pensamentos constantes em um só assunto); ou Fogo no Coração que Consome o Yin e Vazio de Sangue do Coração (Insônia), poderá ser fundamental no direcionamento efetivo do tratamento a pacientes em estágio avançado do processo de stress.

Recomenda-se que a Medicina Tradicional Chinesa faça parte do arsenal terapêutico utilizado por profissionais da saúde que rotineiramente atendem pacientes com desordens orgânicas e psíquicas, cuja base de desequilíbrio é o stress. Este conhecimento deve ser empregado tanto na aplicação das técnicas tradicionais, como para o encaminhamento a profissionais especializados nestas técnicas. Dessa forma, tornar-se-á a saúde integrativa uma das fontes de promoção da qualidade de vida e bem-estar dos pacientes.

\section{AGRADECIMENTOS}

Aos professores do Instituto Superior de Ciências da Saúde (INCISA/BA).

\section{REFERÊNCIAS}

1. Sadir MA, Bignotto MM, Lipp MEN. Stress e qualidade de vida: influência de algumas variáveis pessoais. Paidéia. 2010;20(45):73-81. http://dx.doi.org/10.1590/S0103-863X2010000100010

2. Oliveira-Monteiro NR, Aznar-Farias M, Nava CA, Nascimento JOG, Montesano FT, Spadari-Bratfisch RC. Estresse, competência e problemas psicológicos de adolescentes estudantes. Arq Bras Ciênc Saúde. 2012;37(1):23-9.

http://dx.doi.org/10.7322/abcs.v37i1.45
3. Selye HA. Syndrome produced by diverse nocuous agents. 1936 J Neuropsychiatry Clin Neurosci. 1998;10(2):230-1. http://dx.doi.org/10.1176/jnp.10.2.230a

4. Reis ALPP, Fernandes SRP, Gomes AF. Estresse e fatores psicossociais. Psicol Cienc Prof. 2010;30(4):712-25. http://dx.doi.org/10.1590/S1414-98932010000400004

5. Selye HA. Stress without distress. Filadélfia: Lippincott; 1974. p.97. 
6. Almeida NDV. Considerations concerning the incidence of the stress in professional drivers. Rev Psicol. 2010;1(1):75-84.

7. Lipp MEN, Guevara AJH. Validação empírica do Inventário de Sintomas de Stress (ISS). Estud Psicol. 1994;11(3):43-9.

8. Lipp MN, Malagris LN. O stress emocional e seu tratamento. In: Bernard Range. São Paulo: Artes Medicas; 2001.

9. Zanelli JC. Processos psicossociais, bem-estar e estresse na aposentadoria. Rev Psicol Organ Trab. 2012;12(3):329-40.

10. Lipp MEN, Sassi L, Batista I. Stress ocupacional na equipe cirúrgica. Cad Pesquisa. 1997;3(1-2):57-64.

11. Quick, JC. Líderes saudáveis, organizações saudáveis: prevenção primária e efeitos positivos da competência emocional. In: Rossi AM, Perrewé PL, Sauter SL, editor. Stress e qualidade de vida no trabalho: perspectivas atuais da saúde ocupacional. São Paulo: Atlas; 2007. p.139-55.

12. Santos AFO, Cardoso CL. Profissionais de saúde mental: estresse, enfrentamento e qualidade de vida. Psic Teor Pesq. 2010;26(3):543-48

13. Andrade PS, Cardoso TAO. Prazer e dor na docência: revisão bibliográfica sobre a Síndrome de Burnout. Saúde Soc. 2012;21(1):129-40

http://dx.doi.org/10.1590/S0104-12902012000100013

14 Kovaleski DF, Bressan A. A Síndrome de Burnout em profissionais de saúde. Saúde Transf Soc. 2012;3(2):107-13.

15. Maciocia G. Diagnóstico na Medicina Chinesa - um Guia Geral. São Paulo: Roca; 2005.

16. Doria MCS, Lipp MEN, Silva DF. O uso da acupuntura na sintomatologia do stress. Psicol Cienc Prof. 2012;32(1):34-51. http://dx.doi.org/10.1590/S1414-98932012000100004

17. Fernandes F. Gerenciamento do stress: técnicas eficazes do oriente e do ocidente. São Paulo: Ícone; 2012. p. 23.
18. World Health Organization (WHO). Acupuncture: review and analysis of reports on controlled clinical trials. Geneva: WHO; 2003.

19. Camelo SHH, Angerami ELS. Sintomas de estresse nos trabalhadores atuantes em cinco núcleos de saúde da família. Rev Latino-Am Enfermagem. 2004;12(1):14-21. http://dx.doi.org/10.1590/S0104-11692004000100003

20. Wottrich SH, Ávila CM, Machado CC, Goldmeier S, Dillenburg D, Kuhl CP, et al. Gênero e manifestação de stress em hipertensos. Estud Psicol (Campinas). 2011;28(1):27-34. http://dx.doi.org/10.1590/S0103-166X2011000100003

21. Maciocia G. Os fundamentos da medicina chinesa. São Paulo: Roca; 2014.

22. Cordeiro AT. Acupuntura. São Paulo: Ensaio; 1994.

23. Requena Y. Acupuntura e psicologia. São Paulo: Andrei; 1990

24. Ross J. Sistemas de Órgãos e Vísceras da Medicina Tradicional Chinesa. São Paulo: Roca; 2003.

25. Ross J. Combinação de pontos de acupuntura: a chave para o êxito clínico. São Paulo: Roca; 2003.

26. Schwartz FP. Análise do comportamento dos descritores biomecânicos e eletromiográficos de superfície em exercício resistido por dinamometria isocinética com produção de fadiga. Tese (Doutorado) - Faculdade de Tecnologia da Universidade de Brasília, Brasília, 2010. p. 85-7.

27. Hicks A, Hicks J, Mole P. Acupuntura constitucional dos cinco elementos. São Paulo Roca; 2007.

28. Robaina JR, Lopes CS, Rotenberg L, Faerstein E, Fischer FM, Moreno CRC, et al. Eventos de vida produtores de estresse e queixas de insônia entre auxiliares de enfermagem de um hospital universitário no Rio de Janeiro: estudo Pró-Saúde. Rev Bras Epidemiol. 2009;12(3):501-9. http://dx.doi.org/10.1590/S1415-790X2009000300018 